\author{
KSENIA KUZMINYKH \\ Georg-August-Universität Göttingen \\ kkuzmin@gwdg.de \\ ORCID: 0000-0003-0744-4010
}

\title{
Jugendliterarische Werke im interkulturellen, medienintegrativen Litertaturunterricht
}

\author{
Juvenile books in intercultural, media integrated \\ literature lessons
}

\begin{abstract}
The article starts with a discussion of the essential theories of reading and focuses on different models of understanding. In the next step, based on fragments taken from a novel by $M$. Zusak, the article develops an integrative literary and language teaching model in intercultural school lessons of literature in a way which stimulates a highly functional analytical, receptive and productive handling of language semantics and performance. It demonstrates the combination of literature and film as a possibility for successful literary, language and media learning.
\end{abstract}

KEYWORDS: reading, media, literary and language teaching, intercultural literary, language and media learning, multi-cultural-learning, juvenile books, young-adult books.

SCHLÜSSELWORTE: Lesen, Medien, Literatur- und Sprachunterricht, interkulturelle Literatur, Sprachen- und Medienlernen, multi-kulturelles Lernen, jugendliterarische Werke.

\section{LESEN IM MEHRSPRACHIGEN UNTERRICHT}

Lesen ist für eine erfolgreiche Bildungskarriere sowie für die beruflichen Perspektiven von einer herausragenden Bedeutung und ein sensibler Bereich, wie nationale und internationale Vergleichs- und zahlreiche empirische Forschungsstudien zum Leseverständnis von Schülerinnen und Schüler mit deutscher und einer anderen sprachlichen und kulturellen Herkunft belegen (Baumert 2001). 
Beim Lesen entnimmt die lesende Person dem Text keine Bedeutung. Der Text stellt vielmehr eine Datenbasis für die vorwissensgeleitete mentale Modellkonstruktion, für die Evaluation des konstruierten mentalen Modells und für eine etwaige Revision des generierten Sinnentwurfs dar. Er dient somit als ein Auslöser für mentale Prozesse. Der Leser konstruiert die Bedeutung anhand des Textes unter Rückgriff auf sein sprachliches, textuelles, sozio-emotionales und literarisches, kommunikatives, kulturelles, fachliches, thematisch relevantes und allgemeines Vorwissen. Dieses, aber auch Zielsetzungen, Intentionen, Rezeptionshaltungen, Motive und Erwartungen des Lesers sowie seine Fähigkeit, das Vorwissen heranzuziehen und Inferenzen $\mathrm{zu}$ bilden, determinieren das Sinnkonstruktionsergebnis wesentlich. Der Text im Kopf des Lesers ist demnach sowohl vom Rezipienten als auch vom Produkt (dem Text) abhängig und bildet ein "Text-Rezipienten-Amalgam" (Belgrad, Fingerhut 1998: 6). Die geschriebenen sprachlichen Zeichen initiieren mentale Konstruktionsprozesse, die teils von der externen Textinformation und teils von der internen (im semantischen Gedächtnis gespeicherten) Vorwissensinformation angeleitet werden. Diese Konstruktionsprozesse führen zum Aufbau einer mentalen Repräsentation des im Text beschriebenen Sachverhalts. Das wird subjektiv als Erfassen der Textbedeutung erlebt. Diese Vorstellung wie auch die prinzipielle Unabgeschlossenheit des Deutungsprozesses in hermeneutischer Tradition (Gadamer 1990) bedeuten jedoch nicht, im Sinne eines radikalen Konstruktivismus, dass beliebig viele individualpsychologische Lesearten und Deutungsmöglichkeiten eines Textes existierten, dass er einen Anlass für das selbstreferenzielle Phantasieren liefert, auch wenn die Vielschichtigkeit, Polyvalenz und Ambiguität von literarischen Texten als ein wesentliches Merkmal der Literatur davon unberührt bleiben. Damit ist auch nicht die Reduktion auf eine richtige und mögliche Leseart gemeint. Im schulischen Kontext ist das in der Regel die Auslegung der Lehrperson. Den Texten, auch solchen, die als Literatur gelten, ist ein Widerstandspotenzial inhärent. Dieses lässt sich durch die Zugehörigkeit der Leser (trotz ihrer Individualität) zu einer soziokulturellen Gemeinschaft mit sich etablierten, jedoch auch veränderbaren Deutungstraditionen erklären (Korte 2002). D. h. Texte werden, auch in didaktischer Perspektive, in einen soziokulturellen Zusammenhang eingeordnet. Andererseits erfordert die Text-Leser-Relation ein Gleichgewicht in der Spannung zwischen der Leser- und der Textperspektive. Die Deutungshypothesen sind somit am Text zu belegen, mit Argumenten zu stützen und nur solche mit einem höheren Geltungsgrad bleiben bestehen, werden akzeptiert, können jedoch durch Argumente mit einem noch höheren Wahrscheinlichkeitsgrad entkräftet werden. Dies setzt wiederum das Verstehen auf allen Ebenen voraus: Oberflächenebene, propositionale Ebene, Modell-, Kommunikations- und 
Genreebene (Kintsch 1998, Schnotz 2006). Diese Aufteilung wird lediglich aus heuristischen Zwecken vorgenommen. Die Ebenen überlappen sich im Leseprozess.

Die Oberflächenrepräsentation ist instabil und nicht semantisch, aber sie bildet ein Fundament für die Bildung der darauf aufbauenden höheren Repräsentationen. Die Repräsentation der Textoberfläche ist ohne das Textverstehen möglich. Dieses Phänomen kann bei Lernenden beobachtet werden, wenn Fachtexte oder Definitionen auswendig gelernt werden (Leisen 2013). Es tritt aber auch bei kompetenten Lesern auf, wenn sie Texte in fremden Sprachen, die einem flachen Schriftsystem wie Finnisch oder Japanisch angehören, fehlerlos lesen können, ohne sie allerdings mangels der Sprachenkenntnis zu verstehen. Für zweisprachiges Lesen sind darüber hinaus auf der Ebene der Oberflächenrepräsentation eine geringere Leseflüssigkeit aufgrund von kürzeren Saccaden und längeren Fixationen kennzeichnend. Die kurzen Augensprünge beschränken den Sichtwortschatz. Dies beeinträchtigt eine schnelle(re) Wortekennung (Ehlers 2008).

Bei der propositionalen Repräsentation handelt es sich um das Verstehen des semantischen Gehalts der Aussage und somit um ein rudimentäres Textverständnis. Der Rezipient entschlüsselt Propositionen: hypothetische komplexe Symbole, die sich aus einem Relationssymbol (einem Prädikat) und aus Symbolen für Entitäten (Argumenten) konstituieren. Eine propositionale Repräsentation ermöglicht, den semantischen Gehalt zu verstehen, ohne jedoch das mentale Modell des Dargelegten konstruieren zu können. Bei den Lernenden mit einer anderen sprachlichen Herkunft, aber nicht nur in dieser Zielgruppe, lässt sich dieses Phänomen bei der Rezeption von Texten beobachten, in denen es um fremde kulturelle Phänomene geht. In diesem Fall beziehen sich die Inferenzen auf unterschiedliche Konzepte. Mentale Bilder werden durch andere Assoziationen und Komponenten geprägt, bspw. Weihnachten und das Neue Jahr rufen bei Vertretern verschiedener Kulturen divergierende Vorstellungen. Eine ähnliche Situation vollzieht sich bei der Lektüre von spezifischen Fachtexten, in denen lexikalischen Einheiten eine fachspezifische Bedeutung zukommt. Die Semantik kann gegebenenfalls entschlüsselt werden, aber der (globale) Sinn der Aussage bleibt nicht generierbar. Ein weiteres Beispiel ist die Rezeption von Sätzen oder Texten, in denen es um unmögliche, mit der Alltagslogik nicht konforme Sachverhalte geht, wie z. B. *Farblose grüne Ideen schlafen wütend (Chomsky 1957). Alle Wörter dieses Satzes sind einem deutsche Sprache beherrschenden Rezipienten bekannt, dennoch ergibt er keinen Sinn.

Bei dem mentalen Modell handelt es sich um eine analoge, vorwissensgeleitete Repräsentation des im Text beschriebenen Gegenstands, um eine Vorstellung, ein inneres Bild (Johnson-Laird 1983). Die Konstruktion einer 
mentalen Repräsentation hat von vornherein ganzheitliche Eigenschaften. Manchmal können einzelne Wörter zum Aktivieren einer Vorstellung und somit zum Generieren eines Situationsmodells führen, ohne dass der gesamte Text gelesen und alle Wörter entschlüsselt worden sind. Beobachten lässt sich das bspw. beim Lesen von mathematischen Textaufgaben, wenn bestimmte Schlüsselwörter / verbal cues das Aktiveren vom mathematischen konzeptuellen und prozeduralen Wissen in einem structure-mapping-process in Gang setzen. Weitere Beispiele sind Gefühlserinnerungen, die sogenannten emotion memories, die durch konkrete Anknüpfungspunkte im fiktionalen Text hervorgerufen werden. Der Leser durchlebt sie erneut oder distanziert sich kathartisch von ihnen. Ferner können es emotionale Schemata - formulae of emotion - sein, die analoge Gefühlsmuster beim Leser in Gang setzten (Oatley 1994). Die Rekurrenz auf Gefühle mittels emotionaler Schemata ist insofern von Bedeutung, als ,herausgelesene' Gefühle intensiver bearbeitet und erweitert werden können, ohne dass die lesende Person mit Sanktionen oder Konsequenzen für die in der fiktiven Welt erlebten Erfahrungen rechnen muss.

Die Genreebene impliziert das Wissen über die Makrostruktur des Textes, d.h. über die prototypischen diskursiven Muster bzw. Textsorten. Das Wissen über die makrostrukturellen Besonderheiten wirkt in dreifachen Perspektive unterstützend: bei der Herstellung der globalen Kohärenz, bei dem Aufbau einer bestimmten Erwartungshaltung sowie bei der Konstruktion des mentalen Situationsmodells. Für mehrsprachige Lernende können, in Abhängigkeit von der literarischen Sozialisation, bestimmte Textprototypen, bspw. Märchen, Kurzgeschichten, Kriminalromane, journalistische Berichte oder Zeitungsartikel, Comics durch die Bekanntheit der Textstruktur bei der Rezeption und Produktion von Texten hilfreich sein. Allerdings sollten die spezifischen kulturellen Muster nicht vernachlässigt werden (Kuzminykh 2014).

Die Repräsentation der Kommunikationsebene, in der das Situationsmodell aufgebaut wird, beeinflusst das Leseverstehen ebenfalls. Dies erfolgt, wenn der unmittelbare Kontext der Disambiguierung und der Revision von generierten Sinnentwürfen des Gelesenen beiträgt.

Die Schwierigkeiten im Bereich des Leseverstehens stehen somit in einer unmittelbaren Dependenz zu den Sprachenkenntnissen, allgemeinen (kulturellen, konzeptuellen) Wissensbeständen und dem Textwissen (Ehlers 2008; Ahrenholz 2008; Kniffka, Siebert-Ott 2007).

Die zentrale Annahme der nachfolgenden Überlegungen zum Lese- und Literaturunterricht im mehrsprachigen Unterricht besteht darin, dass mehrsprachige, medienintegrativ ausgerichtete Lernarrangements $\mathrm{zu}$ literarischen Werken eine motivierende Wirkung entfalten. Die Motivation betrifft 
folgende Elemente: den Leselernprozess, sprachlich-kulturelle und strukturelle Erkundungsarbeit am Text, die Förderung von sprachlich-literarischen Fähigkeiten und Fertigkeiten, das verstehende / verständigungsorientierte Interpretieren und den Spracherwerb. Darüber hinaus ist eine lerneffiziente Wirkung anzunehmen. Ferner wird eine Chance für vertiefende literarästhetische Prozesse eröffnet sowie das Erleben von Gratifikationen des Literarischen begünstigt. Veranschaulicht wird das Konzept an exemplarischen Ausschnitten aus dem Werk von Markus Zusak (2008c) Die Bücherdiebin.

\section{MEHRSPRACHIGER LITERATURUNTERRICHT}

Es herrscht mittlerweile ein Konsens, dass Herkunftssprachen der Schülerinnen und Schüler eine wichtige Rolle beim Erwerb einer weiteren Sprache spielen (Krifka 2014; Colombo-Scheffold 2010). Der Literatur kommt in einem mehrsprachigen Deutschunterricht eine nicht weniger geringe Rolle zu. Sie ist ein wichtiger Bestandteil der kulturellen Betätigung einer Gemeinschaft, ein kultureller Kommentar. Sie greift das Vorfindliche auf, pointiert, kritisiert, lehnt sich daran an, distanziert sich davon, akzentuiert und nimmt neue Perspektivierungen vor, verarbeitet es neu und misst schließlich Bedeutungen zu, ohne dass Prinzipien der Ambiguität und Polyvalenz davon berührt werden. D. h. Literatur wird als ein Deutungsangebot, als eine Sichtweise von der Realität bzw. eine Reaktion darauf, als ein Modell rezipiert, nicht als eine Deutung bzw. Realität per se. Der Literatur, besser den Texten, die als solche gelten, wird eine bestimmte Qualität zugeschrieben. Dabei wird oft auf die Ästhetik und Poezität, auf language of performance (Iser 1976), aber auch auf Symptomatik rekurriert (Freise 2006). Schließlich kann Literatur als ein Kommunikationssystem aufgefasst werden, bei dem ein bestimmtes Rezeptionsmodus - literary mode - eingenommen wird (Zwaan 1996). Dies geschieht auch bei der Rezeption von fiktionalen Filmen im Gegensatz zum documentary mode, in dem man eher dazu geneigt ist, das Rezipierte als eine Deutung wahrzunehmen.

Aus dieser Perspektive soll eine grenzüberschreitende Geschichte des Überlebens und der Resilienz in den Blick genommen werden, die international und medial als Verfilmung (Percival 2013) und als Hörbuch (Aljinović 2008) rezipiert und sowohl von Jugendlichen als auch von Erwachsenen gelesen wird. Als weitere Aspekte für die Wahl des Romans fungieren: kulturgeschichtliche Bedeutung, Dichte und Aspektfülle, kontinuierlicher Spannungsaufbau sowie originelle Darstellungsweise, die das Interesse für poetologische Fragen zu wecken vermag. 


\section{DIE BÜCHERDIEBIN VON MARKUS ZUSAK IM MEHRSPRACHIGEN LESE- UND LITERATURUNTERRICHT}

Der Roman von Markus Zusak Die Bücherdiebin steht stellvertretend für eine zeitgeschichtliche Jugendliteratur und kann zu der geschichtserzählenden Literatur gezählt werden. Er widersetzt sich der These einer Kollektivschuld sowie der des Wohlergehens der Arbeiter in der Zeit des Nationalsozialismus. Darüber hinaus bietet er den Leserinnen und Leser „ein tröstendes, versöhnliches Bild des Todes an" (Dauer 2012: 500).

Aus der Perspektive des personalisierten Todes wird die Geschichte von Liesel Meminger, einem neunjährigen Mädchen, das während der Zeit zwischen 1939-1943 von ihrer Mutter zu den Pflegeeltern Hans und Rosa Hubermann nach Molching, einem kleinen (fiktiven) Ort, in Bayern, gebracht wird, erzählt. Die Mutter ist verarmt und kann nicht länger für Liesel und ihren sechsjährigen Bruder Werner, der noch während der strapaziösen Zugfahrt zu dem neuen Aufenthaltsort vom allgegenwärtigen Tod aufgesucht wird, sorgen. Die in der Himmelstraße 33 lebenden Pflegeeltern gehören nicht der gehobenen Schicht an. Rosa Hubermann verdient ihren Lebensunterhalt als Wäscherin, doch werden die Aufträge mit dem Fortschreiten des Krieges rarer und bleiben schließlich aus. Hans Hubermann arbeitet als Anstreicher. Abends spielt er Akkordeon gegen die Inhumanität, Brutalität, Grausamkeit und Sinnlosigkeit des Krieges. Für Liesel ist „der Klang des Akkordeons außerdem ein Ausdruck von Sicherheit. Vom heiligten Tag" (Zusak 2008c: 44). Hans Hubermann weigert sich vehement, der NSDAP beizutreten. Er (er)kennt die Bedrohung, die von der Partei ausgeht und ihre Gräueltaten, dennoch oder gerade deswegen nimmt er Max Vandenberd, einen jungen jüdischen Mann, dessen Vater er im Ersten Weltkrieg kennen und schätzen lernte, und dem er sich verpflichtet fühlt, im Keller seines Hauses auf. Die potenziellen, brutalen Folgen dieser großherzigen Tat sind ihm bewusst wie auch die Konsequenzen, die von seiner spontanen Hilfsaktion - einem Akt der Menschlichkeit -, einem entkräfteten KZ-Insassen ein Stück Brot zu reichen, ausgehen. Zwar kommt es zu keiner unmittelbaren Verhaftung, aber für den Familienvater bleibt nur eine ausweglose, unbarmherzige Alternative, seine Familie zu beschützen, indem er sie verlässt.

Hans kümmert sich liebevoll um Liesel, hilft ihr, Alpträume zu überwinden, vermittelt ihr das Gefühl, willkommen und geliebt zu sein, und bringt ihr das Lesen bei. In Besitz des ersten Buches Handbuch für Totengräber kam das Mädchen nach der Beerdigung ihres Bruders (Zusak 2008c: 44). Liesel hob das vom Lehrling des Totengräbers fallengelassene Büchlein auf, aber sie gab es ihm nicht zurück. Sie behielt es als ein Symbol der Nähe. Da- 
bei „war es ohne Bedeutung, wovon das Buch handelte. Wichtig war nur, was es ihr bedeutete: Das letzte Beisammensein mit ihrem Bruder. Das letzte Beisammensein mit ihrer Mutter "das letzte Bindeglied zu ihm“ (Zusak 2008c: 44). Liesel kann zu diesem Zeitpunkt noch nicht lesen: „Sie starrte die Buchstaben auf dem Einband an und berührte die bedrückten Seiten, ohne eine Vorstellung davon zu haben, was da geschrieben stand" (Zusak 2008c: 44) und später: „Dann las er [Hans Hubermann] den Titel laut vor, mit äußersten Konzentration: „Handbuch für Totengräber'. „So heißt es also, dachte Liesel“ (Zusak 2008c: 72).

Während einer Bücherverbrennung zu Hitlers Geburtstag, zu dem der Pflegevater und Pflegetochter, um den Schein nach außen zu wahren, hingehen, entwendet Liesel ihr zweites Buch Schulterzucken. Sie bringt dadurch ihren Pflegevater auf die für Max rettende Idee, Mein Kampf als ,Ausweis' zu benutzen. In Liesels Besitz sind bereits Faust, Der Hund, Der Leuchtturm, viele weitere werden folgen, denn ihr Verlangen, Bücher zu lesen und zu besitzen, ist unstillbar und steigert sich im Laufe der Handlung, wenngleich sie wählerisch ist: ",sie hatte eigentlich nicht wirklich das Verlangen, die Bücher zu stehlen, die in der Schule gelesen werden" (Zusak 2008c: 101). Es geht ihr um Eskapismus, um die Ästhetik der Wörter sowie um Gratifikationen des Literarischen.

Nach dem mühsamen Einleben in der Himmelstraße 33 findet Liesel Geborgenheit, Freundschaften und Liebe. Rosa Hubermanns Ton ist rau, derb und garstig: „Wenn sie redete, dann schimpfte sie. Sie könnte sich in der Küche mit der ganzen Welt anlegen.“ (Zusak 2008c: 47) „Sie machte Hackfleisch aus Liesel." (Zusak 2008c: 63), aber sogar ihre Lieblingsbezeichnungen "Saukerl" oder "Saumensch" sind in bestimmten Kontexten liebevoll gemeint. Liesels Freund, Rudi Steiner, übernimmt das in seinem an Liesel gewidmeten Brief, indem er sie mit „Liebes Saumensch“ anredet (Zusak 2008c: 107) und später, als er einen Kuss von ihr geschenkt haben möchte (Zusak 2008c: 331). Diese Wendung im seinem Mund ist zärtlicher, liebevoller, verliebter gemeint. Eine interessante Anregung für die Sprachbetrachtung bietet in der deutschen Fassung die Anrede selbst: Es sollte heißen lieber Saumensch. Sowohl an dieser als auch an vielen weiteren Stellen im englischen Original verwendet Zusak deutsche Wörter (Zusak 2005: 102; 8). Die deutschen Wendungen werden in den angeschauten übersetzten Fassungen beibehalten.

Zwischen Max Vandenberd, dem jüdischen Faustkämpfer, und Liesel entsteht eine tiefe Freundschaft, denn ihnen sind ihre Albträume und Liebe $\mathrm{zu}$ Worten, zu den in Büchern dargestellten, fremden, friedlichen, utopischen Welten gemeinsam. Sie wurden zusammengeschweißt durch das stille Ansammeln von Wörtern (Zusak 2008c: 272). Beide können der Anzie- 
hungskraft der Literatur nicht widerstehen. Max fertigt auf übermalten Blättern aus Mein Kampf den Comic Der Überstehmann an und arbeitet an einem weiteren, in seinem eigenen Wortlaut, Anti-Hitler-Buch (Zusak 2008c: 282). Die literalen Fähigkeiten von Hans Hubermann erlauben ihm, von der Front nach Hause zurückzukehren. Auch Liesel beginnt ihre Geschichte im Keller niederzuschreiben.

\section{SPRACHLICH-STRUKTURELLE ARBEIT AM TEXT}

In einer mehrsprachigen Klasse bietet sich die Parallellektüre an. Aus der Perspektive verschiedener Grade von Sprachkompetenz wird auf den Text in verschiedenen Sprachen geblickt. Als Anlass für die sprachlichstrukturelle Arbeit am Text kann gleich der Beginn des Romans genommen werden. Der Tod, als erzählende Instanz, stellt sich mit ungewöhnlich positiven Eigenschaften vor:

I am in all truthfulness attempting to be cheerful about this whole topic, though most people find themselves hindered in believing me, no matter my protestations. Please, trust me. I most definitely can be cheerful. I can be amiable. Agreeable. Affable. And that's only the A's. Just don't ask me to be nice. Nice has nothing to do with me (Zusak 2005: 3). I am not violent. I am not malicious. I am a result (Zusak 2005: 7).

Ich bin nach Kräften bemüht, dieser ganzen Angelegenheit eine fröhliche Seite $\mathrm{zu}$ verleihen, aber die meisten Menschen haben einen tief sitzenden Widerwillen, der es ihnen unmöglich mach, mir zu glauben, so sehr ich auch versuche, sie davon zu überzeugen. Bitte glaubt mir: Ich kann wirklich fröhlich sein. Ich kann angenehm sein. Amüsant. Achtsam. Andächtig. Und das sind nur die Eigenschaften mit dem Buchstaben , $\mathrm{A}^{\prime}$. Nur bitte verlangt nicht von mit, nett zu sein. Nett zu sein ist mit völlig fremd." (Zusak 2008c: 9) (...) Ich bin nicht gewalttätig. Ich bin nicht bösartig. Ich bin das Ergebnis (Zusak 2008c: 13).

Ни капли не кривлю душой: я стараюсь подходить к этой теме легко, хотя большинство людей отказывается мне верить, сколько бы я ни возмущался. Прошу вас, поверьте. Я ещё как умею быть лёгким. Умею быть дружелюбным. Доброжелательным. Душевным. И это на одну букву Д. Вот только не просите меня быть милым (Зусак 2009: 9). Это не ко мне. Я не свирепый. Я не злой. Я итог (Зусак 2009: 12).

Sincramente, me esfuerzo por tartar el tema con tranquilidad, pero a casi todo el mundo le cuesta creerme, por más que yo proteste. Por favour, confia en mí. De verdad, pedo ser alegre. Amable, agradable, afable ... Y eso sólo son las parablas que empiezan por "a”. Pero mo me pidas sea simpático, la simpatía no va 
conmigo (Zusak 2007: 8). No soy violenta. No soy perversa. Soy lo que tiene que ser (Zusak 2007: 10).

In tutta sincerità, mi sforzo di prendere la faccenda allegramente, anche se, a dispetto delle mie proteste, la maggior parte delle persone trova difficile credermi. Per favore, fidati di me. Posso davvero essere allegra. Posso essere amabile. Affettuosa. Affabile. E queste sono solo le parole che cominciano per A. Non chiedermi però di essere bella: essere bella non è da me (Zusak 2008a: 2). Non sono violento. Non sono maligno. Sono un risultato (Zusak 2008a: 3).

Çoğu kimse bütün itirazlarıma rağmen bana inanmamakta inat etse de, bu konuyla ilgili dürüst ve neşeli davranmaya çalışıyorum. Bana güvenin lütfen. Gerçekten neşeli olabilirim. Dost canlısı olabilirim. Uyumlu olabilirim. Şefkatli olabilirim. Ama benden nazik olmamı istemeyin. Nezaketin benimle bir ilgisi yok (Zusak 2008d: 7).

Er (der Tod) nennt in der metafiktionalen Einleitung antizipierend die wichtigsten Punkte der Handlung und die Protagonisten:

Es ist eigentlich eine kleine Geschichte, und sie handelt unter anderem von: einem Mädchen, ein paar Worten, einem Akkordeonspieler, ein paar fanatischen Deutschen, einem jüdischen Faustkämpfer und einer ganzen Menge Diebstählen (Zusak 2008c: 12).

It's just a small story really, about amongst other things: a girl, some words, an accordionist, some fanatical Germans, a Jewish fist-fighter and quite a lot of thievery (Zusak 2005: 6).

Недлинная история, в которой, кроме всего прочего, говориться: об одной девочке, о разных словах, об аккордеонисте, о разных фанатичных немцах, о еврейском драчуне и о множестве краж (Зусак 2009: 11)

Diese Passage, wie auch der unten zitierte Ausschnitt mit den Farben fehlen in der spanischen Fassung.

È la storia di una di quei sopravvissuti - un'esperta nell'arte di essere lasciata indietro - che, fra le altre cose, riguarda: una ragazza, qualche parola, un suonatore di fisarmonica, alcuni tedeschi fanatici, un pugile ebreo, e un bel po' di furti (Zusak 2008a: 3)

Aslında diğer birçok şey arasında şunlarla ilgili küçük bir hikâye: bir kız, kelimeler, bir akordeoncu birkaç fanatik Alman, bir Yahudi boksör, ve bolca hirsızlık (Zusak 2009: 7) Ben sadece adilim (Zusak 2008d: 8).

Die Sätze sind vorwiegend kurz, elliptisch, parataktisch. Die Satzkonstruktionen sind einfach. Es sind wörtliche Wiederholungen, die die persuasive Wirkung verstärken und die Eindringlichkeit fördern, zu finden. Adjektive werden in der deutschen Fassung nicht in der attributiven Funktion 
gebraucht, sondern in adverbialer. Dies ist aus der Spracherwerbsperspektive zugänglicher, denn es besteht keine grammatisch bedingte Notwendigkeit, sie in dieser Verwendungsweise zu deklinieren. Dennoch oder gerade aufgrund der Kunst des Weglassens gelingt es dem Autor und den Übersetzern, spannende, rätselhafte, mysteriöse Atmosphäre zu kreieren und dem Tod, als Erzähler, pointiert einen leichten ironisierenden Ton zu verleihen, ihn in einem anderen Blickwinkel, als ein anteilnehmendes, charmantes, sein eigenes Tun überwachendes, von Fehlern nicht gefeites Wesen [allerdings nicht in der türkischen Übersetzung, in der der Tod sich als Profi bezeichnet (Zusak 2009: 8)], als einen feinen Menschenkenner erscheinen zu lassen. Es entsteht Spannung und Neugier auf den Fortgang der Geschichte. Auch im Film zeigt er sich als Liesels treuer Gefährte, wenngleich die detaillierte Beschreibung der ersten Begegnung mit den Mitteln des Visuellen lediglich eine lakonische Bemerkung zulässt: „Sie hat mich berührt" (Percival 2013: 00.01.49). Der Film transportiert die inhaltliche Komplexität der Narration anders: Dem Filmrezipienten helfen visuelle und akustische Indizien, solche wie Kameraperspektive und -bewegung, Musik und Montage, die Sympathie des Todes Liesel gegenüber zu entschlüsseln.

Der Imperativ ist ein wiederholendes Phänomen und sollte keine Schwierigkeiten bereiten. Die Verwendung von bitte, please, por favor, per favore, lüften in der deutschen, der englischen, spanischen, italienischen und türkischen Sprache charakterisiert den Tod als höflich. Im Russischen wird zwar auf das Höflichkeitspartikel пожалуйста verzichtet, dafür redet der Erzähler seine Leser in der Höflichkeitsformel Sie an. In anderen Sprachen wird der Leser geduzt. Lediglich in der englischen Sprache lässt sich an der Anredeform nicht erkennen, ob geduzt oder gesiezt wird.

Anhand dieser Stelle können sowohl die Wortschatzarbeit, das Sprachund Grammatiklernen - der Wegfall des Kopula-Verbs sein in der russischen Sprache in einem grammatisch vollständigen Satz, der Verzicht auf Personalpronomen in der spanischen und der italienischen Sprachen, Agglutination im Türkischen, Verwendung von Höflichkeitspartikeln, aber auch ähnliche und unterschiedliche Schriftzeichen des lateinischen und kyrillischen Alphabets, Sonderzeichen des Türkischen, entlehnte Wörter und ihre Herkunft - mit Sprachaufmerksamkeit und literaturwissenschaftlicher Erkundungsarbeit am Text verbunden werden. Man kann ein Gespräch über kulturelle Vorstellungen, die dem Tod in den Herkunftskulturen und -sprachen der Schülerinnen und Schüler zugeschrieben werden, initiieren. Teils wird die Wahrnehmung von den Titelseiten des Buches, die im Fall von Bücherdiebin eine gewisse Variabilität aufweisen, geleitet: Die englische Version zeigt den Tod androgyn, wie auch die türkische. Auf dem deutschen Cover ist eine Tendenz zur Männlichkeit zu erkennen, auf der Umschlagseite der 
russischen Übersetzung ist der Tod mit einer schmalen Taille gezeichnet, was seine Feminität betont. Spanische und italienische Varianten verzichten auf die Abbildung des Todes auf dem Buchumschlag. Es folgt in der spanischen La ladrona de libros auf der zweiten Seite die gleiche stark verkleinerte Zeichnung von der tanzenden Liesel und dem Tod, die auch auf dem deutschen Cover zu sehen ist. Im Italienischen ist ein Mädchen mit Büchern abgebildet.

Allein die Zuordnung zu einem bestimmten grammatischen Geschlecht, falls dies in den Herkunftssprachen vorkommt, birgt ein hohes Lernpotenzial, über sprachliche Differenzen und Ähnlichkeiten sowie über kulturelle Vorstellungen, die dadurch determiniert werden, zu reflektieren. Darüber hinaus lassen sich das Konzept der Artikel und Kategorien der Determination und Indetermination fokussieren. Diese stellen gerade für die artikellosen Sprachen eine Herausforderung dar. Im Deutschen ist es der Tod, in der russischen Sprache heißt es смерть (feminin) wie auch in der spanischen Sprache la muerte, im Italienischen la morte, in Türkischen ölüm. Entsprechend sind die mentalen Bilder der Rezipientinnen und Rezipienten von der Geschlechtsdarstellung geprägt und lassen sich mit entsprechenden Eigenschaften, einem maskulinen oder einem femininen Wesen verbinden. Möglicherweise sind diese sprachlich-kulturell geprägten Konstrukte mit der Darstellung des Todes im Roman nicht konform oder weisen sogar in eine entgegengesetzte Richtung: brutal, gewalttätig, schonungslos, bösartig, bedrohlich, gemein, hinterlistig und hinterhältig, unfair und ungerecht. Entsprechend sind die Sinnkonstrukte durch diese Komponenten und Assoziationen determiniert. Kontrastierend dazu kann das im Laufe der Narration ergänztes Erscheinungsbild des Todes stehen - kein Totenschädelgesicht, kein Kapuzenmantel, keine Sense (Zusak 2008c: 335). Die Leser der russischeren Übersetzung werden über die Verwendung des Todes als ein Maskulinum staunen bzw. irritiert sein. Es kommt zu einem (logischen) Kohärenzbruch - die Illustration suggeriert eine weiblich erzählende Instanz смерть -, die von sich jedoch als von einem Mann spricht. Der Titel der russischen Variante, der sich aus einem Adjektiv im attributiven Gebrauch und einem Substantiv zusammensetzt, wird im grammatischen Geschlecht nicht angepasst - The book thief / Книжный bор -, auch wenn es sich hierbei um eine Diebin handelt und in der russischen Sprache, im Gegensatz zum Englischen, die Möglichkeit besteht, das grammatische Genus am Substantiv zu markieren. Erst im Prolog erfährt der Leser, dass der Bücherdieb eine Diebin ist. Das Wort die Diebin wird als ein Diminutiv - воришка - verwendet, das auch das Geschlecht nicht erkennen lässt. Dieses wird an dem begleitenden, deklinierten Adjektiv deutlich. Das Diminutiv offenbart die Sympathie des Erzählers der Hauptfigur gegenüber und verdeutlich gleichzeitig, dass diese 
Tat keinen großen Schaden anrichtet. In der englischen Sprache gibt es bei Substantiven keine Kategorie des Genus, daher lautet der Titel in dieser Sprache The book thief. In der spanischen Sprache sorgt die Verwendung des femininen Artikels la für die Klarheit in Bezug auf das grammatische Geschlecht. Im Italienischen wird das Femininum am Substantiv und durch Artikel - la bambina - markiert. Bemerkenswert ist die wörtliche Übersetzung des italienischen Titels - La bambina che salvava i libri - Das kleine Mädchen, das die Bücher gerettet hat. Es werden nicht nur die Wertschätzung der rettenden Tat (salva - retten), sondern die Bewunderung des kleinen Mädchens zum Ausdruck gebracht.

Ferner ist die Verwendung von Adjektiven in den Sprachen vom Interesse - sind es in der deutschen, in der englischen und in der spanischen sowie in der italienischen Variante Ausdrücke, die mit dem Buchstaben $A$ beginnen, entsprechend dem ersten Buchstaben des Alphabets, was eine Allusion auf spätere Ereignisse der Narration darstellt, stößt der russische Leser auf Adjektive mit dem Buchstaben $D$, die übersetzt nicht vollständig mit den von Zusak im englischen Original verwendeten Attributen übereinstimmen. Darüber hinaus geht hier noch in der deutschen, englischen, spanischen und italienischen Sprache vorzufindende Anspielung auf den Anfang des Alphabets verloren. In der türkischen Übersetzung fehlt sie.

Von Zusak und von Übersetztern angepasst in der Reihenfolge: Englisch, Deutsch, Russisch, Spanisch, Italienisch, Türkisch: Dabei wird in der deutschen Fassung ein Adjektiv mehr verwendet.

cheerful - fröhlich, лёгкий, alegre, allegra, neşeli amiable - angenehm / amüsant, дружелюбный, amable, amabile, dost agreeable - achtsam, доброжелательный, agradable, affettuosa, uyumlu affable - andächtig, душевный, afable, affabile, şefkatli

Übersetzt in einem Wörterbuch, ohne Anpassung an die Idee des Autors, die Eigenschaften mit dem Anfangsbuchstaben des Alphabets beginnen zu lassen

cheerful - heiter, весёлый, alegre, allegro, neşeli

amiable - liebenswürdig, любезный, amable, amabile, sevimli agreeable - wohltätig, выражающий согласие, agradable, piacevole, hoş affable - leutselig, приветливый, afable, affabile, nazik

Bemerkenswert ist die Ähnlichkeit des Spanischen, des Italienischen und des Englischen. Dies kann als ein Anlass, sich mit der Sprachentwicklung und Sprachverwandtschaft zu beschäftigen, genommen werden. Darüber hinaus kann die Leistung der Übersetzer gewürdigt werden wie auch die Tatsache, dass es keine vollständige Übereinstimmung, d.h. keine formale und semantische Äquivalenz, zwischen der Ausgangs- und der Zielsprache 
gibt. Das Lesen bedeutet somit nicht das Übersetzen in die Erstsprache. Sprache, ihre Arbitrarität und Konventionalität werden mit dieser Art der Wortschatzarbeit als kulturelle Erscheinung bewusst. Literatur bietet mit ihrer reichen Sprache hierfür besondere Möglichkeiten: Einige Zeilen weiter beschreibt der Erzähler die Farben des Tages: „Wachsgelb, regenbesprühtes Blau. Schlammige Dunkelheit" (Zusak 2008c: 11). / "Waxy yellows, cloudspat blues. Murky darkness“ (Zusak 2005: 5). / „Восковатые жёлтые, синие с облачными плевками. Грязные сумраки“ (Zusak 2009: 10). / "Gialli cerei, azzurri plumbei. Tenebrosa oscurità." (Zusak 2008c: 3) / „Bulutumsu maviler, mat sarılar. Çamurlu karanlıklar" (Zusak 2009: 9). Ergiebig sind im Sinne der Sprachlichkeit des literarischen Lernens folgende Aspekte: eine bewusste Wahrnehmung der Wertigkeit der Wörter, Sätze und Aussagen im Textsystem, Untersuchung der Metaphorik und Wirkung der stilistischen Mittel, bspw. der differierenden Farbnuancen und kontrastierende Wortschatzarbeit. Sinnvoll ist eine Aufforderung zu eigenen Beobachtungen und Beschreibungen des Wahrgenommen mit erweiterten vor- und nachgestellten Attributen. Die Schüler können auf diese Weise für die funktionale Kraft der Sprache, wie sie sich besonders in literaturästhetischer Verwendung zeigt, sensibilisiert werden. Somit wird die Arbeit an Komponenten literarischer Analyse einschließlich der Verwendung ausgewählter Elemente aus dem literaturwissenschaftlichen Instrumentarium, Grammatik bei der komplexen Attribuierung, Lexik und kreatives, paralleles Schreiben sinnvoll in das unterrichtliche Geschehen eingebunden. Aus der didaktischen Perspektive ist einer vergleichenden Rezeption ein hohes Potenzial beizumessen: Sie ermöglicht nicht nur eine kulturabhängige Lektüre, sondern fördert die Erfahrung der Alterität, der ästhetischen Differenz und trägt der Erweiterung des kulturellen, des literarischen und des ästhetischen Horizonts bei. Die einzelnen Lexeme können von den Erstsprechern in den jeweiligen Sprachkontext eingebunden und den anderen Schülerinnen und Schüler in der Klasse erläutert werden. Auf diese Weise lässt sich das isolierende Üben lexikalischer und grammatischer Phänomene vermeiden und der Ansatz des impliziten grammatischen Lernens verwirklichen (Feilke, Tophinke 2016). Dabei erfolgt das Sprachenlernen kontextualisiert, d.h. man lernt nicht zunächst Wörter und grammatische Strukturen, die anschließend angewendet werden, und wesentlich später, wenn überhaupt, den ästhetischen Gebrauch dieser Mittel. Von Anbeginn des Lernprozesses werden die Schülerinnen und Schüler dazu ermutigt, sprachaktiv vorzugehen und Mut zur (literarischen) Kommunikation und Lektüre in einer fremden Sprache und zum interkulturellen mehrsprachigen ästhetischen Interagieren aufzubringen. Sie eigenen sich Strategien des Lese- und Sprachlernens an. Ferner können die erarbeiteten Eigenschaften aus den jeweiligen Ausschnitten übertragen und 
erweitert bzw. vertieft werden. Liesel erlernt das Alphabet, indem zu jedem Buchstaben ein Gegenstand oder ein Lebewesen gefunden wird (Zusak 2008c: 75 ff.). Analog dazu können die Schülerinnen und Schüler ihr persönliches Alphabet mit Charaktereigenschaften erstellen. Dies wäre eine sinnvolle dem Ansatz reading-to-write folgende, propädeutische Arbeit für das anschließende Verfassen von Beschreibungen im Deutschunterricht.

Aus der literaturwissenschaftlichen Sicht lässt sich an diesen Auszügen die ungewöhnliche Erzählinstanz zum Gegensand der unterrichtlichen Betrachtung machen sowie der Aufbau des Romans. Nimmt man das Schema zur Erkundung des Erzähldiskurses und zum Verfassen von eigenen Erzählungen als Grundlage (Kuzminykh 2017), lassen sich an den ausgewählten Auszügen bereits einige wichtige Aspekte auf der thematischen Ebene (das Was des Erzählens) und stilistischen Ebene (das Wie der Narration) klären. Es handelt sich hierbei um Figuren und Handlung, Erzählzeit und erzählte Zeit, Orte und Räume, Objekte und den Zustand. Herausfinden lassen sich das Thema, Motive und Botschaft, Intention, Erzähler, Stil und Form. Es handelt sich hierbei um Elemente, die den Schülerinnen und Schüler in Abhängigkeit von der Enkulturation und der literarischen / medialen Sozialisation aus Herkunftsländern bekannt sein können. Wenn es in den Kulturen Narrationen gibt, was mit einer sehr hohen Wahrscheinlichkeit der Fall ist, denn Erzählen (weltweit) ist ein Versuch, die Kontingenz des Lebens im Akt der Narration zu bewältigen, dann lassen sich die in den Erzählungen genannten Komponenten aufspüren. Darüber hinaus ist eine antizipierende Lektüre nach der Rezeption von Titeln in mehreren Sprachen, von Illustrationen auf den Umschlagseiten der Bücher sowie der Cover von Verfilmungen denkbar und vielversprechend, denn auch hier werden kultur- und adressatenspezifische Akzentuierungen vorgenommen.

Die transkulturelle Thematik des Zweiten Weltkrieges und somit der historische Rahmen könnten ebenfalls als bekannt angenommen werden. Beides eröffnet jedoch gleichzeitig Möglichkeiten für eine tiefergehende individuelle, intensive Beschäftigung mit dem historischen Hintergrund des Romans bzw. grundsätzlich mit dem Thema Flucht und politischen Entwicklungen im 21. Jahrhundert. Im Mittelpunkt der Handlung stehen die kindlichen Erfahrungen in einer nicht jüdischen Arbeiterfamilie während der Zeit des Nationalsozialismus, der Hunger, die Armut, die Arbeitslosigkeit, das Leid der einzelnen Gruppen, ohne dass es gegeneinander aufgewogen wird, und das Wissen um den Umgang mit jüdischer Bevölkerung - der Alltag zwischen Angst, Anpassung und der Rebellion im Kleinen, ohne eine Überhöhung, Idealisierung und Glorifizierung positiver Helferfiguren, ohne heldenhafte, starke Widerstandskämpfer, aber auch ohne Stereotypisierung. Das Böse wird weitgehend undifferenziert durch die Person Hitler darge- 
stellt. Die unterschiedlichen Figuren im Roman bilden im Syntagma der Narration ein Netz an Korrespondenzen - Opfer, Täter, Mitläufer und Helfer während der Zeit des Nationalsozialismus.

Ein weiteres Thema ist die Bedeutung des Lesenlernens, der Literatur und Sprache im Roman, das wiederum eine transkulturelle Relevanz hat. Liesel kann nicht lesen und muss es mühsam lernen. Sie trifft auf sehr ähnliche Schwierigkeiten auf allen Ebenen im Leseverstehensvorgang, auf die Schülerinnen und Schüler mit einer anderen sprachlichen und kulturellen Herkunft im Literaturunterricht stoßen. Sie erliest mühsam die Wörter: „Es waren nur drei - ,der', , die' und ,das'. Auf der ganzen Seite standen insgesamt etwa zweihundert Worte" (Zusak 2008c: 75). Sie versucht den Propositionen Sinn zu geben, stellt sich mentale Bilder vor, konstruiert Situationsmodelle des Gelesenen und das an einem Thema (Handbuch für Totengräber, Zusak 2008c: 44), das fremder nicht sein kann. Schließlich wagt sie Wörter in ihrer ästhetischen Funktion und Wirkung zunächst aufgemuntert von Max, später um ihn aufzumuntern, zu gebrauchen (Zusak 2008c: 272 ff.).

Ihrer Unfähigkeit zu lesen, wird mit Intoleranz seitens der neuen Mitschülerinnen und Mitschüler begegnet. Dennoch gibt sie nicht auf: „Sie sah sich bereits als ein zehnjähriges Wortewunder. Wenn es bloß so einfach wäre" (Zusak 2008c: 73). Die Rolle der Literatur und ihr Bedeutungspotenzial lassen sich an ausgewählten Passagen untersuchen (Zusak 2008c: 71-77 Liesel lernt lesen; 149-152 in der Bibliothek der Frau des Bürgermeisters, Zusak 2008c: 140 Bedrohung für die Köpfe und Herzen des deutschen Volkes); und vielleicht sind den Schülerinnen und Schülern Beispiele und Personen aus den Herkunftsländern bekannt, die trotz aller Widrigkeiten und Hindernisse vergleichbare Situationen meisterten. Für den russischen Kontext ist der Name von Lomonosov, einem brillanten Schriftsteller, herausragenden Wissenschaftler, Reformer der russischen Sprache, Verfasser der russischen Grammatik und Gründer der Moskauer Universität, die seinen Namen trägt, zu nennen, der zu Fuß im rauen Winter mehrere Tausend Kilometer von Archangelsk nach Moskau zurücklegte, um an der Geistlichen Akademie studieren zu dürfen. Darüber hinaus lernte er ohne fremde Hilfe lesen, schreiben und lateinische Sprache. Thematisieren lassen sich die Rolle des Lesens, die oft den Kinderbüchern inhärent ist (Karg, Kuzminykh 2014), die Bedeutung und Mühseligkeit des Schreibens - für einen Brief an ihre leibliche Mutter brauchte Liesel drei Stunden und sechs Anläufe (Zusak 2008c: 109) -, die Einflusskraft der Sprache und die eigenen Erfahrungen der Lernenden damit. Im Roman wird die Macht der Sprache in vielfältigen Facetten evident: in der Sammlung von Gedanken, Die Worteschüttlerin, die Max Liesel nach seiner Flucht hinterlässt (Zusak 2008c: 476); in der Rhetorik von Hitler, die ihm zur Machtposition, aber auch zur Zerstörung des deutschen 
Volkes verhalf; in den Schimpftriaden von Rosa Hubermann, bald zärtlich und liebevoll, bald wörtlich aufzufassen; in den Worten von der Frau des Bürgermeisters, die Rosa kündigt; zusammengefasst in das Buch Mein Kamp, dessen Lektüre als Tarnung Max vor Kontrollen rettet; Worte als Trost- und Hoffnungsspender auf dem Weg nach Dachau im Gefangenenmarsch; als faszinierende Kraft, die Max im Keller am Leben hält; als eine Ablenkung im Luftschutzbunker, wenn Liesel den Nachbarn vorliest; als Antipode zur Sinnlosigkeit des Krieges und schließlich als Lebensretter im Bombenhagel vom 7. Oktober von 1943, währenddessen Liesel an ihrem Buch arbeitet. Verstehen der Bedeutung und der Wirkung der Sprache, wäre eine wichtige Aufgabe des mehrsprachigen Literaturunterrichts. Ergiebig sind die Untersuchungen zur Wirkung der Sprache in Fächerkombinationen - Politik / Werte und Normen / Ethik / Religion.

\section{MEDIENINTEGRATIVER LESE- UND LITERATURNTERRICHT}

Ein weiterer Aspekt für einen mehrsprachigen, medienintegrativen Unterricht ist die Arbeit mit der Verfilmung des Romans, die ebenfalls internationale Rezeption erfuhr. Englische, deutsche, spanische und türkische Verfilmungen erschienen 2013, russische einige Monate später, im Januar 2014. Darüber hinaus gibt es ein Hörbuch (Zusak 2008b). Allen medialen Repräsentationen des Romans ist zwar die Kongruenz in der Grundstruktur inhärent, aber sie bieten in der für sie spezifischen, ästhetisch-literarischen Diversität unterschiedliche Interpretationen dieser fiktionalen Vorlage oft mit differierenden Komplexitätsgraden, Akzentuierungen und Perspektivierungen, Anpassungen an die potenziellen Rezipienten und an ihre Rezeptionserfahrungen an. Dadurch werden der Möglichkeitssinn, Ambiguität und Vielschichtigkeit der Literatur evident. Die ästhetische Breite, die Multiperspektivierung, Pluralismus und Dissoziation der einzelnen medialen Komponenten und medialen Interpretationen bergen ein hohes Potenzial für das Lesen, für literar-ästhetisches und sprachliches Lernen und eröffnen bedeutsame rezeptologische Chancen. Die unterrichtliche Arbeit kann auf eine doppelte Weise verwirklicht werden - entweder in einem sequenziellen Medienwechsel oder als ein transformativer Prozess in Sinne von schemaverändernden Rezeptionserfahrungen. Bei der zuletzt genannten Möglichkeit werden die Abschnitte aus dem Medienverbund in einer vorab festgelegten Reihenfolge rezipiert, so dass schließlich ein virtueller Text, eine Geschichte, im Kopf während des Prozesses der intermedialen Kohärenzbildung entsteht. Gerade für mehrsprachigen Literaturunterricht ist entscheidend, dass die Unterschiede zwischen den einzelnen, für die unterrichtliche 
Thematisierung ausgewählten Versionen nicht zu gravierend sind und die potenziellen Kohärenzbrüche durch die Hinführungs- und Übergangsmoderationen überwunden werden können. Sind die Unterschiede zwischen den einzelnen Fassungen zu stark ausgeprägt, kann es dazu kommen, dass die Rezipientin oder der Rezipient sich nicht auskennt und unmotiviert ist, sich weiter mit dem literarischen Gegenstand auseinanderzusetzten. Bei sehr ähnlichen Fassungen wird die Bedeutung als eine sich ausbreitende Verarbeitung von cues bearbeitet. Diese organisieren den von Neugierde- und Spannungshypothesen geleiteten Inferenzprozess.

Für das verstehende Lesen kann die vorangehende Filmrezeption, die wie ein advanced organizer eingesetzt wird, eine positive Wirkung haben. Es können sich Vertrautheit mit den Motiven, mit den Figuren, mit den Handlungskonstellationen und dem Handlungsrahmen der literarischen Narration einstellen. Dies initiiert eine kontrastierende, eine vergleichende und ggf. eine wiederholende Rezeption. Visuelle Darstellungen können die Konstruktion des mentalen Situationsmodells des literarischen Kommunikats erleichtern. Sie schaffen somit eine Grundlage sowohl für eine Bedeutungskonstruktion und folglich für das Verstehen als auch für das sprachliche und literar-ästhetische Lernen. Dies ist wiederum ein Fundament für die Erweiterung, Evaluation, Revision und Neustrukturierung von frames, scripts und schemata. Man kann diesen Vorgang als zyklisch beschreiben: Im Zentrum des Sinnkonstruktionsprozesses befindet sich der Text - das Was und das Wie der Narration. Um dieses Zentrum organisieren sich die unterrichtlichen Praktiken. Auf der Seite des Lernangebots sind es Elemente, die den Schülerinnen und Schülern in ihren Strukturen und Anspruchsniveaus zugänglich sind, die ihnen vertraut oder fremd sind, und die sie teils kennen und teils nicht. Auf der Seite der unterrichtlichen Praktiken sind es erworbenes Wissen, Schemata und Erfahrungen sowie Wahrnehmung des Angebots, das dynamisch mit den Elementen verbunden ist. Die durch die den literarischen Medienverbund repräsentierte Objektseite ist aufgrund der strukturellen Spezifika in besonderer Weise in der Lage, auf der Ebene der Handlung (Was des Erzählens) und der Darstellung (Wie des Erzählens) Bekanntes und Vertrautes anzubieten, das wahrnehmungs- und wissensgeleitete Sinnbildungsprozesse ermöglicht. Hinzu kommt, dass nicht nur die unterschiedlichen Modi des Erzählens vertreten sind (verbalsprachlich schriftlich, visuell, akustisch, verbalsprachlich mündlich, audiovisuell), sondern auch verschiedene inhaltliche Muster, die die narrativen Schwerpunkte unterschiedlich arrangieren, ausdehnen und perspektiveren. Dies wiederum geschieht auf der Basis verschiedener erzählerischer Stile und unterschiedlich ausgeprägter ästhetischer Komplexität, so dass prinzipiell die Möglichkeit besteht, dass sich die bestehenden Schemata verändern und 
auch die Wahrnehmung in veränderten Weise geleitet und herausgefordert wird.

Die sprachliche Komplexität wird mit den spezifischen Mitteln des audiovisuellen, multimodalen Mediums Film dargestellt. Diese können, im Sinne der im kultursoziologischen Diskurs kritisierten Egalität der visuellen Medien, bis zu einem bestimmten Grad von allen Rezipientinnen und Rezipienten aufgenommen werden. Die Sinngenerierung erfolgt vor dem Hintergrund je individueller Wahrnehmung und Wissensdispositionen von Rezipientinnen und Rezipienten und stellt grundsätzlich ein Fundament für den Prozess der Sinnbildung dar. Es ist nur wichtig, dass es nicht dabei belassen bleibt. Das gibt dem mehrsprachigen medienverbindenden Literaturunterricht eine Chance.

Es handelt sich sowohl bei dem oben zitierten Ausschnitt als auch bei dem gesamten Film um eine illustrierende Literaturadoption. Dies ermöglicht eine parallele Medienrezeption und erleichtert den Zugang zum Text. In aller Exemplarität sei dies an dem oben zitierten Ausschnitt aus dem Film Die Bücherdiebin (Persival 2013, 00.00.01 - 00.03.00) veranschaulicht: Die Kameraeinstellung in der Maintitel-Sequenz ist eine Totale. Der Filmrezipient sieht weiß, Wolken, Nebel, Winter im regenbesprühten Blau und einen Zug. Dies erleichtert das Verstehen der von Zusak verwendeten Epitheta zur Beschreibung des Tages. Die Rezipienten sehen die Farben. Das Motiv des Zuges ist wiederkehrend für die Filme, die sich mit der Thematik des Nationalsozialismus beschäftigen, und somit intertextuell bedeutsam. Nur um einige Beispiele zu nennen: Steven Spielbergs Schindlers Liste oder Roman Polanskis Der Pianist, Jochen Alexander Freydanks Das Spielzeugland, Mark Hermans Der Junge im gestreiften Pyjama, Christian Duguays Ein Sack voll Murmeln. Anschließend geraten die Fahrgäste während der Kamerafahrt durch den Wagon in den Fokus bis sie auf Liesel stehen bleibt. Das Mädchen wird zunächst in Groß, dann im Detail gezeigt. Ihr Blick richtet sich ihrem Bruder zu und als sie erkennt, dass er nicht mehr lebt, erfolgen ein harter Schnitt und der Szenenwechsel: Es werden die Beerdigung und der erste Diebstahl des Buches gezeigt. Akustisch wird dies durch das schrille Signal und die Geräusche des schnell fahrenden Zuges begleitet. Die sympathische Stimme des Erzählers voller Wärme und Zuneigung, die des Todes, verschwindet. Die von Zusak installierte Erzählinstanz ist allerdings laut dem Drehbuchautor Petroni in der verfilmten Fassung nicht so dominant. Es bedarf einer aufmerksamen Rezeption und einer komplexeren Inferenzbildung, um den Erzähler als Tod zu bestimmen. In einer kombinierenden Rezeption können sich die textuellen und visuellen Informationen ergänzen.

Es wäre jedoch sinnvoll die ausgewählten Passagen nicht lediglich für den Zweck der verstehenden Lektüre zu instrumentalisieren, sondern auch 
zum Gegenstad des ästhetischen Lernens, der visual literacy, zu machen und die medienspezifische Verwendung von Darstellungs- und Effektmöglichkeiten zu beobachten. Darüber hinaus erweitert dies das Vokabular um filmsprachliche Begriffe.

Eine weitere Möglichkeit zur Kommunikation bietet die Thematisierung von Buch- und Filmkritiken oder von Trailern, Klappentexten und Blurbs in den Herkunftsländern der Schülerinnen und Schüler. In Deutschland erfuhr der Roman eine positive Resonanz, während der Film eher auf eine ambivalente, fast ablehnende Haltung stieß. Die Schülerinnen und Schüler können sich den literatur- und filmkritischen Diskurs anschauen und Ausschnitte daraus im Unterricht referieren. Dies wäre ein Betrag zum transkulturellen Lernen.

\section{FAZIT}

Der vorgestellte medienintegrative Entwurf zielt modellhaft auf die metakognitive Dimension des Lernens und des Lesens sowie auf Förderung der sprachlich-kulturellen Kompetenz, da in den erzählten Welten sowohl kulturspezifische als auch kulturübergreifende Komponenten sichtbar werden. Es geht nicht darum, den Roman in seiner Gänze zu lesen, dies wäre im mehrsprachigen Literaturunterricht aufgrund des Umfangs und der Aspektdichte ein arbeits- und zeitintensives, und damit unrealistisches Unterfangen, sondern in aller Exemplarität mit Ausschnitten aus dem Roman in mehreren Sprachen und seiner Verfilmung zu arbeiten. Sprachlernen und literarische Lektüre kommen als genuine kulturelle Komponenten in den Blick. Es geht aber auch um Wissensvermittlung von Traditionen kulturgeschichtlicher Bedeutungszumessungen. Dies ermöglicht das Verstehen von kulturell bedeutsamen Texten und kann die Habituation des Lesens (auch) in der fremden Sprache begünstigen.

\section{LITERATURVERZEICHNIS}

Ahrenholz, B. (2008). Erstsprache - Zweitsprache - Fremdsprache. In: B. Ahrenholz / I. Oomen-Welke (Hrsg.). Deutsch als Zweitsprache. Baltmannsweiler: Schneider, S. 3-16.

Baumert, J. (2001) (Hrsg.). Deutsches PISA-Konsortium. Pisa 2000. Basiskompetenzen von Schülerinnen und Schülern im internationalen Vergleich. Opladen: Leske + Budrich.

Belgrad, J. / Fingerhut, K. (1998). Annäherungen an Literatur im Unterricht. In: J. Belgrad / K. Fingerhut (Hrsg.). Textnahes Lesen. Baltmannsweiler: Schneider, S. 5-14.

Chomsky, N. (1957). Syntactic structures. New York: de Gruyter Mouton.

Colombo-Scheffold, S. (2010). Ausländisch für Deutsche: Sprachen der Kinder - Sprachen im Klassenzimmer. Freiburg: Fillibach. 
Dauer, Y. (2012). Verfolgung, Angst, Tod - und Hoffnung? Der 2. Weltkrieg im Spiegel der Kinder- und Jugendliteratur am Beispiel von Markus Zusaks „The book thief". In: A. Bartl / A. Klinge (Hrsg.). Transitkunst: Studien zu Literatur 1890-2010. Bamberg: Bamberg Press, S. 475-513.

Ehlers, S. (2008). Lesekompetenz in der Zweitsprache. In: B. Ahrenholz (Hrsg.). Deutsch als Zweitsprache. Baltmannsweiler: Schneider, S. 215-227.

Feilke, H. / Tophinke, D. (2016). Grammatisches Lernen. Praxis Deutsch, 256, S. 4-12.

Freise, M. (2006). Vier Weisen nach dem Text zu fragen. In: V. Bockholt / M. Freise / W. Lehfeldt / P. Meyer (Hrsg.). Finis coronat opus. Göttingen: Vandenhoek \& Rupprecht, S. 71-83.

Gadamer, H.-G. (1990). Hermeneutik. Wahrheit und Methode: Grundzüge einer philosophischen Hermeneutik. Tübingen: Mohr Siebeck.

Iser, W. (1976). Der Akt des Lesers. München: Fink.

Johnson-Laird, P.N. (1983). Mental models. Cambridge: CUP.

Karg, I. / Kuzminykh, K. (2014). Sprache und Literatur als Bildungskomponenten. Diskurs, Historie und Empirie. Frankfurt a. M.: Peter Lang.

Kintsch, W. (1998). Comprehension: a paradigm for cognition. Cambridge: CUP.

Kniffka, G. / Siebert-Ott, G. (2007). Deutsch als Zweitsprache. Lehren und lernen. Paderborn: Schöningh.

Korte, H. (2002). „K wie Kanon und Kultur. Kleines Kanonglossar in 25 Stichwörtern.“ In: H.L. Arnold (Hrsg.). Literarische Kanonbildung. München: edition text + kritik, S. 25-38.

Krifka, M. (2014). Das mehrsprachige Klassenzimmer. Berlin: Springer.

Kuzminykh, K. (2014). Comics und graphic novels im multikulturellen Deutschunterricht. Glottodidactica. An International Journal of Applied Lingiustics, XLI (2), S. 75-87.

Kuzminykh. K. (2017). Erzählen im integrativen Deutschunterricht. Glottodidactica. An International Journal of Applied Lingiustics, XLIV (2), S. 75-95.

Leisen, J. (2013). Handbuch Sprachförderung im Fach - Sprachsensibler Fachunterricht in der Praxis. Stuttgart: Klett-Sprachen.

Oatley, K. (1994). A taxonomy of the emotions of literary response and theory of identification in fictional narratives. Poetics, 23, S. 53-74.

Percival, B. (2013). Die Bücherdiebin. Deutschland / USA.

Schnotz, W. (2006). Was geschieht im Kopf des Lesers? Mentale Konstruktionsprozesse beim Textverstehen aus der Sicht der Psychologie und der kognitiven Linguistik. In: H. Blühdorn / E. Breindl / U. Waßner (Hrsg.), Text - Verstehen. Grammatik und darüber hinaus. Berlin: de Gruyter, S. 222-238.

Zusak, M. (2005). The book thief. Sydney: Picador.

Zusak, M. (2007). La ladrona de libros. Barcelona: Lumen.

Zusak, M. (2008a). La bambina che salvava i libri. Milano: Mondolibri.

Zusak, M. (2008b). Die Bücherdiebin. Hörbuch (gesprochen von Boris Aljinović). München: Random House Audio.

Zusak, M. (2008c). Die Bücherdiebin. München: Blanvalet / Random House.

Zusak, M. (2008d). Kitap hırsızını. Istanbul: Selim Yeniçeri.

Зусак, М. (2009). Книжный вор. Москва: Эксмо.

Zwaan, R. (1996). Toward a model of literary comprehension. In: B. Britton / A. Graesser (Hrsg.). Models of understanding text. Mahwah NJ: Erlbaum, S. 241-256.

Received: 31.10.2017; revised: 22.01 .2018 